\title{
How to Think About Driverless Vehicles
}

\author{
Noah J. Goodall
}

\author{
Review of Driverless: Intelligent Cars and the Road Ahead by Hod Lipson and Melba Kurman \\ Cambridge, MA: The MIT Press; 2016 \\ $328 \mathrm{pp} ; \$ 29.95$ \\ ISBN-10: 0262035227 \\ ISBN-13: 978-0262035224
}

Pre-print version. Published in American Journal of Public Health, 109(8), September 2018, pp. 1112-3. Available at: http://dx.doi.org/10.2105/AJPH.2018.304572

It is difficult to overstate how driverless transportation will affect the economy, public health, and personal mobility. The numbers are staggering - $87 \%$ cheaper cab fares, $39 \%$ reduction in trucking costs ${ }^{1}$ saving the industry $\$ 500$ billion each year in the United States, up to 1.3 million lives saved from crashes worldwide ${ }^{2}$ - and it will allow frictionless movement for the young, elderly, and mobility impaired.

Those are just the benefits. The automation of driving may also put 5 million American professional drivers out of work, enable a foreign government to halt traffic via cyberattack, allow police to effortlessly lock down and cordon off a city, or paradoxically increase congestion as individual car travel becomes so cheap and comfortable as to discourage public transit to the point of elimination.

Any other topic with such profound yet near-term impacts would have inspired many books by now, yet driverless technology has only a handful. I suspect the lack of attention given to driverless vehicles is in fact a symptom of its importance - anyone with even passing interest in vehicle automation seems to be working directly on the technology or its regulation. With such a demand for talent, no one has the time to write. A technology with impacts on society that rival splitting the atom for once has the energy and funding of the Manhattan Project.

Fortunately, Hod Lipson and Melba Kurman's Driverless takes on this profound topic with a fascinating and thorough examination of the social and technical issues surrounding vehicle automation.

\section{The Importance of Sensing}

Lipson's background is in machine vision, which becomes evident around Chapter 5 and is absolutely unmistakable in Chapter 10. Machine vision - that is, the ability for a computer to tell the difference between a car and a pedestrian from video stills - is of obvious importance for a self-driving vehicle navigating a busy road. Lipson's expertise leads to the book's best sections, such as the history of machine learning in Chapter 10. Some of the highlights include one of the clearest explanations of a neural network I have come across and the tragic story of a young Frank Rosenblatt and his unfairly maligned Perceptron, an early artificial neural network made of lightbulbs and a few wires that was demonstrated on live television in 1957.

Lipson's background in machine vision is also the book's greatest weakness, as his overfamiliarity with the topic leads to some jargon-heavy passages. Unless one is interested in the Neocognitron's C-cells or the $\mathrm{A}^{*}$ search algorithm, one can safely skip a few pages here and there without losing much context. Lipson also gives far more attention to machine vision, while overlooking the far more useful (at least on today's prototype vehicles) laser scanners. 


\section{Can Wireless Communication Fill the Gaps?}

Video detection, machine vision, laser scanning, and all other ways a vehicle can sense the environment will occasionally miss an object in one of three ways: detection (Is it there?), classification (What is it?), and prediction (What will it do next?). The Tesla that fatally struck the side of a white tractor trailer in Florida had both a detection and classification error, as the car's radar was looking too low for bumpers and missed the elevated truck trailer, while the camera saw the truck but interpreted the white truck as part of the sky. ${ }^{3}$ A minor crash between a Waymo automated vehicle and a bus in 2016 was an error of prediction, as the Waymo vehicle incorrectly assumed that the bus would wait for Waymo to merge into traffic. ${ }^{4}$ These errors can be drastically reduced if vehicles can simply communicate their location (I'm here!), status (I'm a car!), and intentions (I'm about to merge!) directly with nearby vehicles. Fortunately, the US Department of Transportation and major automakers have been working on such a system since the 1990s, currently distinguished as vehicle-to-infrastructure communications or vehicle-to-vehicle communications. Collectively, these are known as connected vehicles.

Lipson and Kurman spend several pages dismissing the US Department of Transportation's emphasis on vehicle-to-vehicle communication, often by unfairly conflating it with vehicle-to-infrastructure communications. It is simply not true that the safety and mobility benefits of wireless communication among vehicles requires a nationwide network of communication devices every mile or two at a cost of $\$ 57 \mid 650$ each. Although this would be nice to have, almost every vehicle-to-infrastructure application can afford a bit of lag and can be accomplished over forthcoming $5 \mathrm{G}$ communication networks by using something resembling an advanced Waze-like app. Even if vehicle-to-infrastructure communication never comes to fruition, vehicles can form ad-hoc vehicle-to-vehicle networks and share information down the line, allowing one vehicle that hits black ice to pass a warning along, vehicle by vehicle, to cars miles behind. At a cost of merely $\$ 300$ per car, mandating that new cars come equipped with vehicle-to-vehicle radios could greatly improve safety of conventional vehicles while nearly eliminating sensor error of automated vehicles.

\section{A Better Metric for Safety}

The book's most novel contribution is an alternative metric for safety. Today, hardware reliability is measured in failures per million miles, and safety as crashes per hundred million miles. These numbers are hard for consumers to grasp and do not allow direct comparison with the alternative, a human driver. Lipson and Kurman propose the metric humansafe, with which an automated vehicle that crashes half as often as a human driver has a humansafe rating of 2 . This is a much-needed metric and is an invaluable tool for educating consumers on the safety of vehicle automation. However, we should be clear that the rating of a sober, attentive, alert driver is a bit higher than humansafe 1, as the average crash rate includes all kinds of crashes preventable with existing technology such as alcohol ignition interlocks, speed limiters, and driver attention-monitoring systems. A discussion of why these safety systems seem unnecessary yet full automation is somehow morally required would be quite valuable.

Lipson and Kurman go a bit further and argue that automated vehicles should be legalized once they reach a humansafe level of 2 . This is fine if (1) it is safer than a sober driver, (2) the vehicle can demonstrate this safety level with statistical significance (driving twice the average human miles between crashes is not enough), (3) the remaining crashes are of equal or lesser severity (one fatal crash is worse than two damage-only crashes), and (4) the remaining crashes do not unfairly target class of road users. On the last point, a major reduction in vehicle-vehicle crashes may not compensate for even a small increase in pedestrian fatalities, especially as pedestrians get no mobility benefits from automated vehicles. 
Driverless provides a much-needed overview of the truly massive social and economic impacts of vehicle automation, with a valuable history of the technology that some readers may not want but probably need. Public health professionals will find it an excellent resource for understanding what will be the greatest safety innovation of the next generation.

\section{References}

1. Torrey WF, Murray D. An analysis of the operational costs of trucking: 2016 update. Minneapolis, MN: American Transportation Research Institute; 2016. Available at: http://atri-online.org/wpcontent/uploads/2016/10/ATRI-Operational-Costs-of-Trucking-2016-09-2016.pdf. Accessed May 3, 2018.

2. World Health Organization. The top 10 causes of death. 2017. Available at: http://www.who.int/newsroom/fact-sheets/detail/the-top-10-causes-of-death. Accessed May 3, 2018.

3. Tesla Inc. A tragic loss. 2016. Available at: https://www.tesla.com/blog/tragic-loss. Accessed May 3, 2018.

4. Harris M. Google accepts blame for its self-driving car causing crash. New Scientist. March 1, 2016. Available at: https://www.newscientist.com/article/2079133-google-accepts-blame-for-its-self-drivingcar-causing-crash. Accessed May 3, 2018. 